BMJ Editorial; holt03003

Technical editor: Liz Payne, epayne@bmj.com

This is the editorial of your article that will appear online; please check the proofs carefully.

General points:

- We work to a strict house style and have to make specialist articles accessible to general readers, including overseas readers whose first language may not be English. Thus changes will inevitably have been made to your manuscript.

- We edit articles for clarity, accuracy, and conciseness.

- We accommodate authors' changes whenever possible, but please keep them to a minimum.

- Please check that all queries to author (indicated by emboldened text in square brackets) have been answered.

\title{
Chest pain in primary care: what happens to the undiagnosed majority?
}

Pain without a diagnosis may signal higher cardiovascular risk

Tim Holt

Senior Clinical Research Fellow

University of Oxford

Nuffield Department of Primary Care Health Sciences

Radcliffe Observatory Quarter

Woodstock Road

Oxford

OX2 6GG

tim.holt@phc.ox.ac.uk

Research, doi:10.1136/bmj.j1194

Acute chest pain is an alarming symptom, and the exclusion of a cardiac or other serious cause is the first priority. Through the process a proportion of patients undergo investigation and in some cases coronary heart disease is confirmed, a definite non-cardiac diagnosis is made, or the cause is unattributed. Assessment of new onset chest pain is typically based on the pattern and characteristics of the pain in the context of background cardiovascular risk. In a linked paper, Jordan and colleagues (doi:10.1136/bmj.j1194) show that most patients are managed without undergoing cardiac investigations. ${ }^{1}$ The unattributed group, whether investigated or not, have a greater risk of myocardial infarction over the next five years than those with a more definite non-cardiac diagnosis, as we might expect. More surprisingly, their volume in terms of patient numbers is sufficient such that more myocardial infarctions occur over the following five years in this group than those labelled with a cardiac cause at 
the outset. The latter are at higher relative risk but are outnumbered as a population by the large group with an unspecified cause. They are also managed very differently, aiming for tight control of vascular risk factors on an indefinite basis. Arguing for more effective assessment in general, the authors suggest that efforts to control the risk of those in the unattributed group are warranted.

The core finding of this paper - that the five year cardiovascular risk of the patients without a diagnosis is in fact substantial - might be explained in two ways. Firstly, the chest pain might in fact be of cardiac origin, but for some reason this escapes recognition in the assessment that follows. In such cases any reassurance is misplaced. Alternatively, there might simply be a statistical association between a tendency to report genuinely non-cardiac pain and increased risk of future vascular events. Confounders might include both physical and mental health factors. In these cases the short term reassurance is valid.

These findings expose a wider dilemma over the justification for intervention (or investigation) in large but relatively low risk populations. For high risk individuals, any risk of harm associated with intervention or investigation may be justified. However, an approach targeting this group alone will be of limited impact if the majority of events occur in the larger lower risk population; a paradox relevant also to primary prevention of cardiovascular disease and first described by the epidemiologist Geoffrey Rose. ${ }^{2} 3$

The study by Jordan and colleagues shows the potential of linking routine data from primary and secondary care sources with disease registers to answer important research questions. Linkage enables a wider capture of events and a more comprehensive understanding of what is happening. Awareness of the processes governing data recording in the respective environments is important. General practitioners in the UK may be confident that a patient has angina before investigation, but they will typically await confirmation before recording an electronically coded diagnosis, as this step places the patient on the coronary heart disease register and removing them is not straightforward if the diagnosis is not confirmed.

Inclusion on the register requires multiple processes for follow-up and ongoing monitoring. Non-specific "symptom” codes are often used at this point, before a formal diagnosis, however confident the clinician may be. The authors used a six month lag to ensure that, if made after investigation, the diagnosis found its way into the record. This enables a meaningful definition of the population without a diagnosis, even though the motivation to record electronically a non-cardiac cause is not always strong. 
What does all this mean for clinical practice? Firstly, new onset of chest pain in an individual may justify more active investigation and less reliance on clinical assessment alone than is currently practised, given these results. Secondly, the study supports the current approach where interpretation and choice of investigations are influenced by the background cardiovascular risk of the individual. Predictive values are determined partly by pretest probability, so interpretation of test results needs to include assessment of background risk to avoid misplaced reassurance. Finally, we might recognise that pain with no attributed cause may be an early signal, or simply a proxy for an increased risk of future myocardial infarction.

The unattributed group, however, represents a diverse clinical taxonomy. In addition to those whose coronary heart disease may have been missed, people likely to be harmed by excessive investigation may be over-represented in this group, such as those with panic disorder. In one study, 25\% of patients with chest pain in an emergency department were found to have panic disorder. ${ }^{4}$ Future research might clarify how we can better assess and meet the individual needs of patients with unattributed chest pain.

Competing interests: I have read and understood the BMJ policy on declaration of interests and declare the following: none.

Provenance and peer review: Commissioned; not peer reviewed. $<$ jrn $>1$ Jordan KP, Timmis A, Croft P, et al. Prognosis of undiagnosed chest pain: linked electronic health record cohort study. BMJ 2017;357:j1194.</jrn>

$<$ jrn>2 Hunt K, Emslie C. Commentary: the prevention paradox in lay epidemiology--Rose revisited. Int J Epidemiol 2001;30:442-6. PubMed doi:10.1093/ije/30.3.442</jrn>

$<\mathrm{jrn}>3$ Rose G. Sick individuals and sick populations. Int J Epidemiol 1985;14:32-8. PubMed doi:10.1093/ije/14.1.32</jrn>

$<$ jrn $>4$ Fleet RP, Dupuis G, Marchand A, Burelle D, Arsenault A, Beitman BD. Panic disorder in emergency department chest pain patients: prevalence, comorbidity, suicidal ideation, and physician recognition. Am J Med 1996;101:371-80. PubMed doi:10.1016/S0002-9343(96)00224-0 $<$ /jrn $>$

The Corresponding Author has the right to grant on behalf of all authors and does grant on behalf of all authors, a worldwide licence to the Publishers and its licensees in perpetuity, in all forms, formats and media (whether known now or created in the future), to i) publish, reproduce, distribute, display and store the Contribution, ii) translate the Contribution into other languages, create adaptations, reprints, include within collections and create summaries, extracts and/or, abstracts of the Contribution, iii) create any other derivative work(s) based on the Contribution, iv) to exploit all subsidiary rights in the Contribution, v) the inclusion of electronic links from the Contribution to third party material where-ever it may be located; and, vi) licence any third party to do any or all of the above. 